\title{
Isolation and Identification of Fungi from fast food restaurants in Langa Bazar
}

\author{
Sewgil Saaduldeen Anwer ${ }^{1}$, Gazang A. Ali² , Chra Z.Hamadamin ${ }^{3}$, Hanan Y. Jaafar ${ }^{4}$ \\ ${ }^{1}$ Department of Clinical Biochemistry, College of Health Science, Erbil, Kurdistan Region, Iraq. \\ ${ }^{2,3,4}$ Department of Medical Microbiology, College of Health Science, Erbil, Kurdistan Region, Iraq.
}

\begin{abstract}
A total of (218) samples from Eleven different foods were processed between October 2016 and February 2017 which include (Tomato, Chicken meat, red meat, falafel, potato, bread, eggplant, cabbage, celery, cucumber and onion). Samples were collected from 4 different fast food restaurants inoculated on Potato dextrose agar and Sabouraud Dextrose Agar. Isolated fungus identified morphologically and microscopically in accordance with standard procedures. Results showed that six fungal genera were associated with the selected fast food restaurants. The isolated fungal genera were Aspergillus sp., Alternaria sp., Mucor sp., Rhizopus sp., Saccharomyces sp., Brettanomyces $s p$. The number of total colonies in October were 236 and in February were 119 and the number of colonies were higher when cultured on Potato dextrose agar than Sabouraud Dextrose Agar. There was variation in the pattern of occurrence of the fungus in fast foods Aspergillus sp. appears to be the most pathogenic fungi that present in the food samples.
\end{abstract}

Keywords- Fungus, Fast food restaurants, Food spoilage.

\section{INTRODUCTION}

Food spoilage is any change in the appearance, smell, or taste of a food product that makes it unacceptable to the consumer. Spoiled food may still be safe to eat, but is generally regarded as unpalatable and will not be purchased or readily consumed. Food spoilage causes losses to producers, distributors, and consumers in the form of reduced quality and quantity and higher prices (1). Another case of spoilage could be that the nutrients (e.g. vitamin content) in the food have deteriorated to the point that the food product no longer meets its declared nutritional value. The time it takes for a food product to reach one of these spoilage conditions is generally termed the product's shelflife potential food spoilage microorganisms include bacteria, fungi (mold and yeast), viruses, and parasites.(2). Action by microorganisms is a common means of food spoilage and the most common cause of foodborne illness.
Microbial spoilage is a major concern for so-called perishable foods such as fresh fruits, vegetables, meats, poultry, fish, bakery products, milk, and juices. Meat and dairy products, with their high nutritional value and the presence of easily metabolized carbohydrates, fats, and proteins provide ideal environments for microbial spoilage (3).

A wide variety of fungi, including species of Rhizopus, Alternaria, Penicillium, Aspergillus, and Botrytis spoil foods. Since fungi grow readily in acidic as well as lowmoisture environments, fruits and breads are more likely to be spoiled by fungi than by bacteria. AspergillusFlavus infects peanuts and other grains, producing aflatoxin, a potent carcinogen monitored by the food and drug Administration (4).

The factors that affect microbial growth in food and constantly the association that develop also determine the nature of spoilage and any health risks posed for convenience they can be divided into four groups Intrinsic factors Intrinsic factors of a food include nutrients, growth factors, and inhibitors or (antimicrobials), water activity, $\mathrm{pH}$, and oxidation-reduction potential. The influence of each factor on growth in a food system the factors are present together and exert effects on microbial growth in combination, either favorably or adversely $(5,6)$. , Extrinsic factors Which include environmental factors like temperature, Relative humidity and gases, Implicate factors Include Specific growth, Synergism, Antagonism and commensalism competition among the microorganism that cause changes of physical and chemical structure of the food and spoiled the food $(7,8,9)$.and Processing factors Include the tools with slaughtering and cutting the water which use in washing, vehicles which are used in transporting, and retail market (8).

Food-borne illness by fungi:

A food-borne illness occurs when a person becomes ill after eating or drinking contaminated foods or beverages. Nothing that we eat or drink is completely pure, and many 
microorganisms live in the foods and beverages that we consume. Microorganisms or microbes are tiny organisms such as bacteria, Fungi, viruses, and parasites that exist in our bodies as well as in plants, animals, food, water, air, and soil. Many of these microbes do us no harm, and some "friendly" ones are necessary for a healthy body, but other microbes can cause infections that lead to serious, or even fatal, illness. Additionally, there are harmful chemicals and other substances in food that can cause a food-borne illness (10).

Fungi include mold and yeasts which are more adaptable to various conditions than other microorganisms. Have high tolerance for acidic condition and more often responsible for food spoilage than for food-borne illness (11).

\section{MATERIAL AND METHODS}

\section{Media preparation:}

Two media can be used, Potato Dextrose Agar (PDA) and Sabourated Dextrose Agar (SDA). They were prepared according to the manufacturer's instructions 39gm (PDA) powder in $1 \mathrm{~L}$ of distilled water for PDA medium. And $62 \mathrm{gm}$ (SDA) powder in $1 \mathrm{~L}$ distilled water for SDA medium , then sterilized and used for fungal cultivation.

\section{Collection of samples and Isolation:}

A total of 218 sample from 11 different foods were obtained from 4 restaurant three times a day respectively ( 9:30 AM, 12:00 PM, 4:30 PM) during the months of October 2016- February 2017. The food samples were taken from restaurant in sterile plastic bags according to Cheesbrough 1984 Direct plate used for culturing the Individual yeast and mold species (figure 2.1) The samples transferred with sterile forceps into Petri dish contain sterilized SDA Saboured dextrose agar and PDA Potato Dextrose Agar and the plates were incubated at $25^{\circ} \mathrm{C}$ for 5 7 days (12)

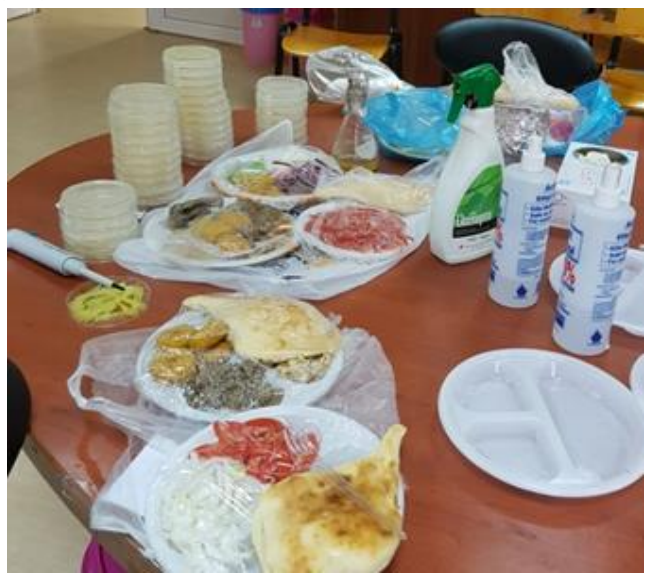

Fig. Sampling and Culturing of fungi on SDA and PDA.

\section{Identification:}

Fungal isolates were transferred to sterilized plates for purification and identification. The grown fungi were placed on a slide, stained with gram stain for yeast identification and lacto phenol cotton blue to detect fungal structures covered with a cover slip, examined under microscope and identified on the basis of their colony morphology and spore characteristics (12).

Macroscopic and microscopic observations were carried out on the cultures. The physical characteristics of the mycelia such as the colour and structure were noted as well as the microscopic characteristics $(13,14)$.

\section{RESULTS}

\subsection{Variation of colony numbers in Fungus during} October:

After collecting the samples and culturing we obtained the colonies and after examining the colony under microscope we obtained some fungal genera that associated with food spoilage. Figure 3.1.showspercentage of colonies isolated from fast food restaurants during October ( 22/10/2016).

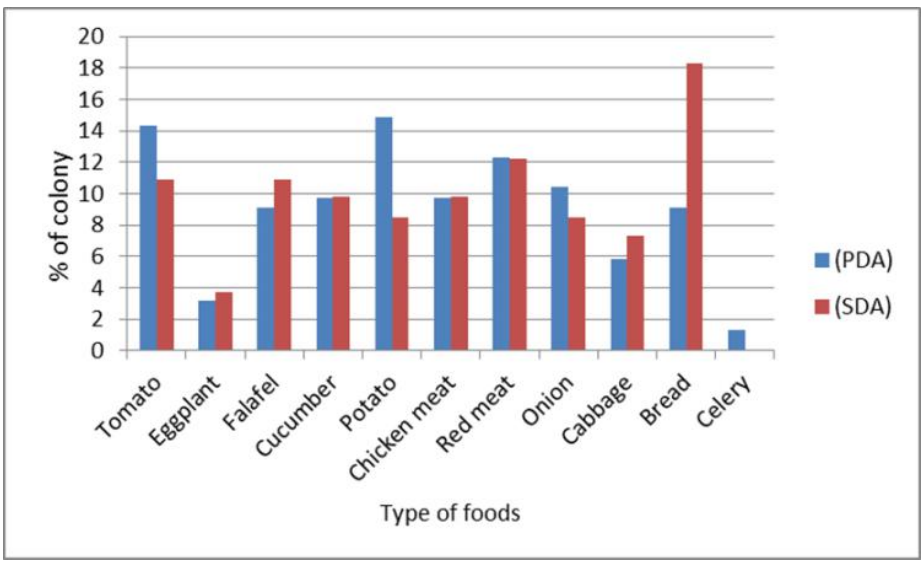

Fig.3.1: Percentage of total colony isolated in food during October in (PDA, SDA).

\subsubsection{Variation of colony of Fungus number during February:}

The number of colony decreased when sample collected and examined by cultivation to obtain colony.figure 3.2 shows the percentage of colonies isolated from fast food restaurant during February (14/2/2017). 


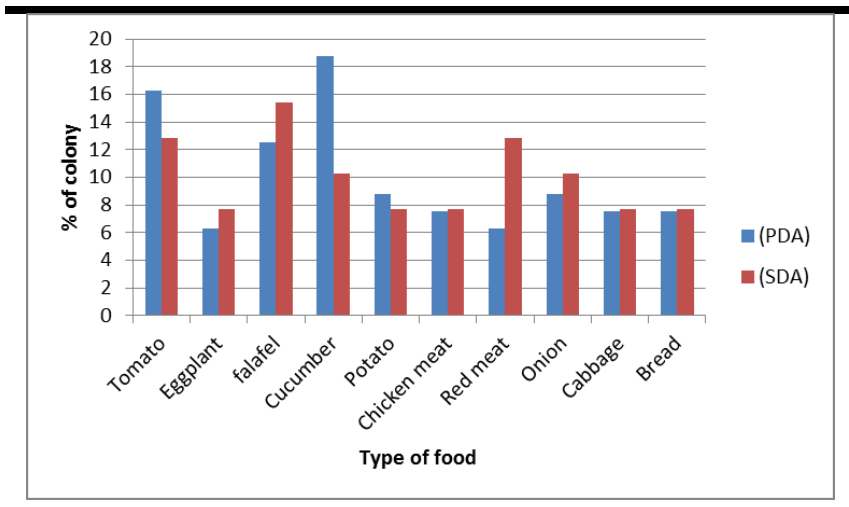

Fig: Percentage of total colony isolated in food during February in (PDA, SDA) medium

\subsection{Detection of Fungus In different foods:}

From a total of (218) collected samples and 11 different foods from different fast food restaurants a total of 6 genera (mold and yeast)

were isolated as shown in the table 3.1

Table.3.1: Occurrence of fungal genera in different food sample.

\begin{tabular}{|l|l|l|}
\hline No. & Food type & Fungal genera \\
\hline $\mathbf{1}$ & Tomato & Alternaria sp. \\
\hline $\mathbf{2}$ & Eggplant & Mucorsp. \\
\hline $\mathbf{3}$ & Falafel & Saccharomyces sp. \\
\hline $\mathbf{4}$ & Cucumber & Aspergillus sp. \\
\hline $\mathbf{5}$ & Potato & Mucor sp. \\
\hline $\mathbf{6}$ & Chicken meat & Aspergillus sp. \\
\hline $\mathbf{7}$ & Red meat & Aspergillus sp. \\
\hline $\mathbf{8}$ & Onion & Aspergillussp. \\
\hline $\mathbf{9}$ & Cabbage & Brettanomycessp. \\
\hline $\mathbf{1 0}$ & Bread & Rhizopus sp. \\
\hline $\mathbf{1 1}$ & Celery & Alternariasp. \\
\hline
\end{tabular}

The major problem associated with fast food is the frequent incidence of contamination .Due to the nature of these foods and their methods of preparation involving extensive handling. The results of the present study revealed that different molds were isolated from 11 different food samples namely eggplant, falafel, chicken meat, red meat, celery, cabbage, potato, tomato, bread and cucumber collected from four locations fast food restaurant in Langa Bazar. The isolated molds were identified as food-borne. The molds include Aspergillus sp., Alternaria sp., Mucor sp., Rhizopus sp. and Saccharomyces sp. and Brettanomyces sp. Several studies have reported similar molds from different snacks and fast food contamination from water, air, storage distribution facilities, environment and human activities researchers $(15,16,17)$. Yassin and his coworkers isolated same fungal strains from restaurant and meat shops in al Samawa (18).

\subsection{Cultural and Microscopic properties of Fungal isolates:}

Microscopically examination for isolates from Cucumber, Red meat, Chicken meat and onion showed that Conidiophores and septate, unbranched hyphae. With a swollen apex (vesicle) .Phialides born on the metulae (biseriate). Conidia in dry chains forming compact columns (columnar) that can be identified as Aspergillus sp. Colonies on PDA agar at $25^{\circ} \mathrm{C}$ within 7 days, and consisting of a dense felt of green conidiophores. (Figure 3.3)

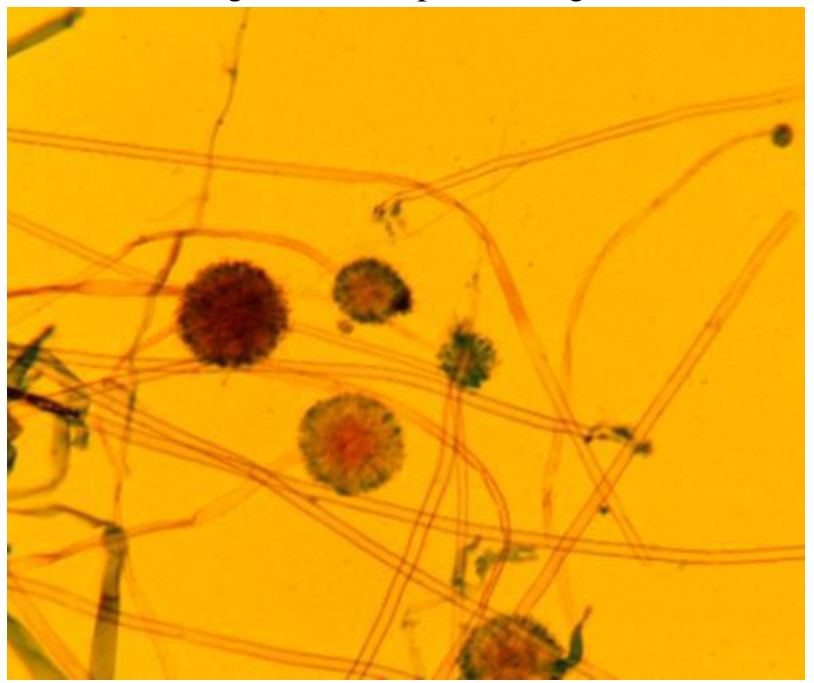

Fig.3.3::Microscopic examination of Aspergillus sp.

Microscopically examination for isolates from falafel .showed that cells quite large, globules to sub globes, reproduce by budding as showed in figure 3.4.and the colonies on PDA agar at $25{ }^{\circ} \mathrm{C}$ within 7 days, and whitish mucous texture that can identified as Saccharomyces sp.

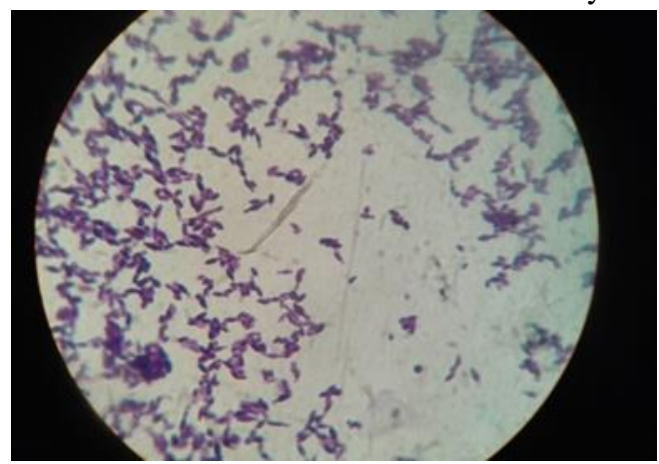

Fig.3.4: Microscopic examination of Saccharomyces sp.

Isolates from cabbage under microscope appears that the cells are olive to cylindrical shaped and Colonies on PDA agar at $25{ }^{\circ} \mathrm{C}$ appear as white small soft cotton textures as 
showed in figure 3.5. and the isolates identified as Brettanomyces sp.

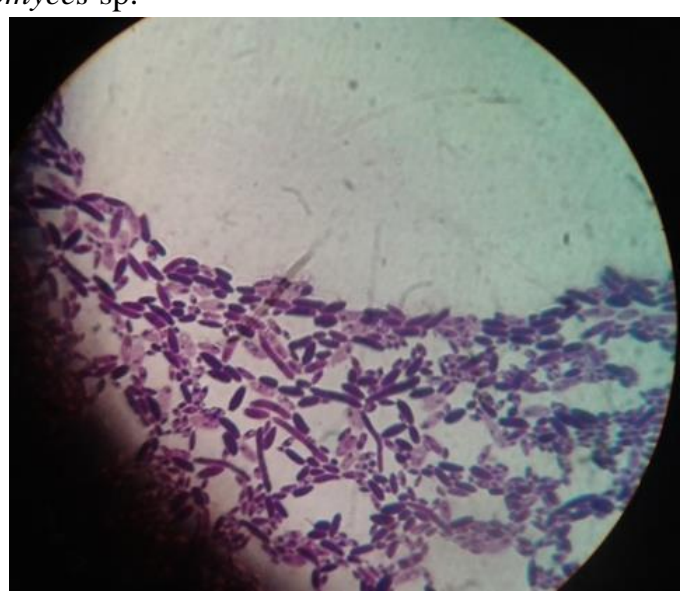

Fig.3.6: Shows microscopic examination of Brettanomyces

sp.

Microscopic features that was used to help in identification of isolates from Bread showed that sporangiospores, sub globes, and irregular in shape with Apophysis and having stolone and Rhizoids and colonies on PDA, was whitish becoming grayish-brown. Figure 3.6. And can be identified as Rhizopus sp.

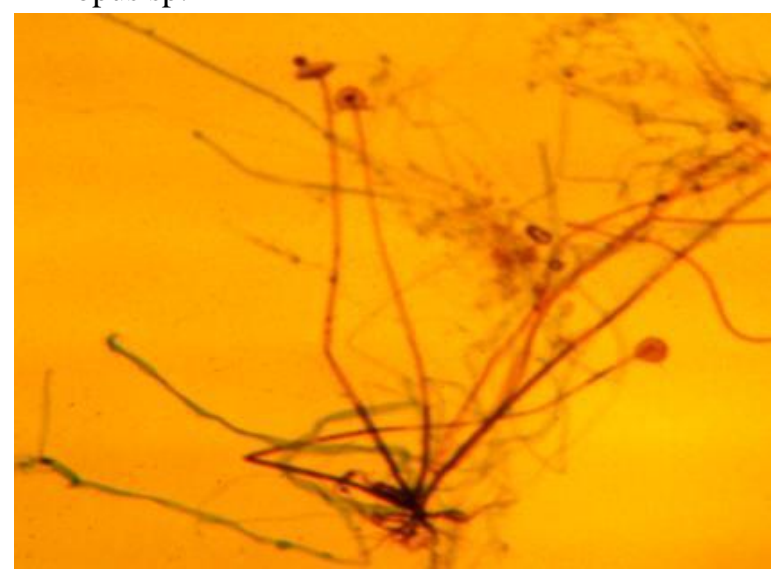

Fig.3.5: Microscopic examination of Rhizopus sp.

When isolate from Eggplant and Potato examined under microscope hayphae was non septate, sporangiophores simple at first, later slightly sympodially branched, with sporangium at terminal ends without apophysis and colonies on $\mathrm{SDA}$ at $25^{\circ} \mathrm{C}$, resemble white-to-gray cotton candy, darkening with time. Fegure 3.7. The isolates can be identified as Mucor sp.

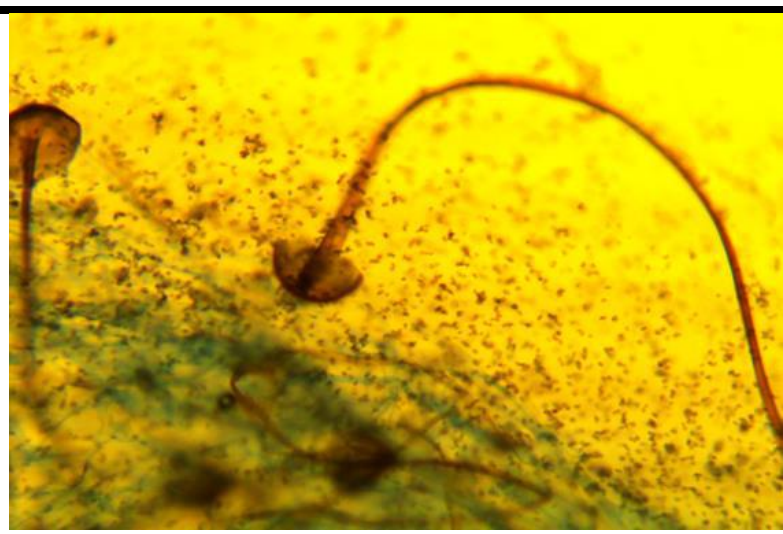

Fig.3.7: Microscopic examination of Mucorsp

Microscopically appearance of isolates from tomato and celery showed that septate branched pale brown to olive brown with secondary conidiophores, conidia in long branched chains. Colonies on PDA at $25^{\circ} \mathrm{C}$ for 7 days, olivaceous-black or greenish black surrounded with white cotton hyphae and is identified asAlternaria sp. ( figure 3.8.).

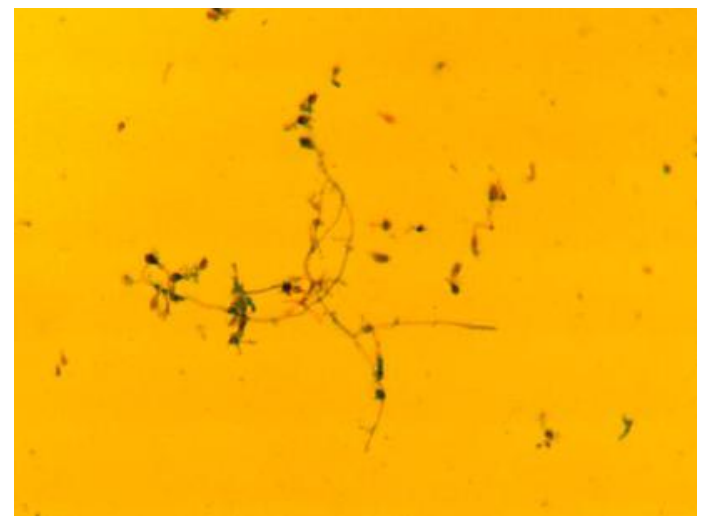

Fig.3.8: Microscopic examination of Alternaria sp.

As showed in figure 3.9: There was no growth of fungus on plate contain PDA that incubate in $25^{\circ} \mathrm{C}$ for 7 days (used as control).

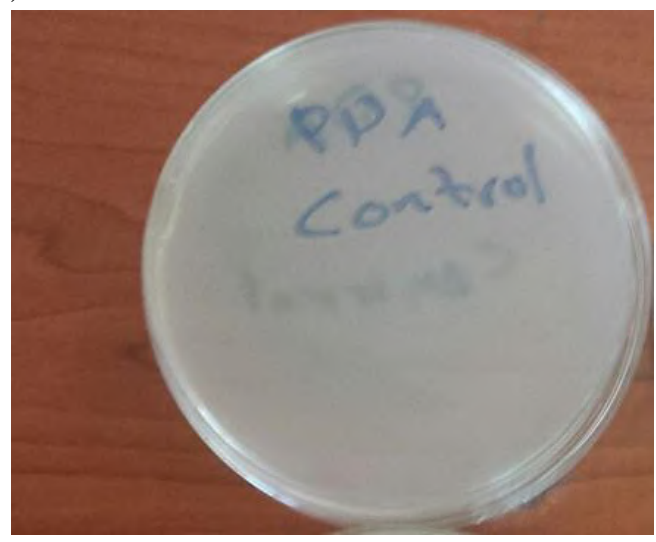

Fig.3.9: PDA medium used as control. 
After collecting the samples and after subculturing them on PDA and SDA the number of colonies that obtained were (96) colonies at 9:30 AM (81) colonies at 12:00 PM and at 4:30 PM (59) colonies as showed in table 3.1 the number of colonies at 9:30 AM were more than other times (12:00 PM and at 4:30 PM) the reason for this result is using previously made foods that is not stored properly at 9:30 AM and using of newly made foods in the noon and after noon.

The number of total colonies in October were 236 and in February were 119 and this return to the fact that the tempreature in month October is higher than in month february and so making and storing the foods in high tempreature and in an inappropriate condition that help to increase number of fungal colony during October.

The number of colonies were higher when cultured on PDA than SDA in spiteof using antibiotic in both PDA and SDA to prevent the growth of bacteria and other microrganism the reason for the result may be due to that PDA is a general purpose media for molds and yeasts. Potato dextrose agar (PDA) may be slightly superior to Sabouraud dextrose agar (SDA) for growing molds since SDA was originally formulated to detect fungi associated with skin infection (19).

Aspergillus sp. was the most spoilage fungi isolated from most type of foods and this result are resemble to the experiments that done by Easa 2010 which isolated different species of Aspergillus from traditional fast foods (20)

As it appear in our result we obtained the growth of different fungi and this may be due to not wearing special fastfood uniform during woking or not using appropriate methods and means to keep the equipments and the surrounding clean also another cause is food processing was in an open place where it is close to sidewalk pedestrians and cars. Also it may be due to cutting the vegitables and meats on the same cutting board or wrapping a broken knife handle with plastic covers and using of expired (sauce, mayonnaise, amba, ketchup).

\section{CONCLUSIONS}

Several types of fungi were isolated from fast food restaurant which cause food spoilage. The number of colonies isolated from fast food restaurants was higher during October because of increase of temperature and number of shoppers. Some food samples showed high number of Colonies during early morning. The result showed there are some pathogenic fungi present in fast food restaurants like (Aspergillus sp., Alternaria sp., Mucor sp., Rhizopus sp., Saccharomyces sp., Brettanomyces sp.) and the number of colonies on PDA was more than SDA.Our suggestion for fast food restaurant isto keep the foods away from contamination and spoilage and this will be by commitment with health rules that deal with restaurants cleanly and proceeding regular tests for restaurants workers and to Study presence or absence $t$ of mycotoxin in the food products.

\section{REFERENCES}

[1] Joanne M, Linda M, and Christopher J. Microbial Growth and Food Spoilage, Microbiology, New York, 2008.7th ed. 1026.

[2] Blackburn W, Clive de W. Introduction and Microbial Spoilage,Food Spoilage Microorganisms 2006. 1-11.

[3] Sperber W. Microbiological Food Spoilage Mechanisms, compendium of the Microbiological Spoilage of Foods and Beverages, New York, 2009. 10.

[4] Eugene W, Denise G C, Evans Roberts, Nancy N and Martha T. Common Spoilage Fungi, Microbiology, 2004 .4th ed. 811.

[5] Adams MR and Moss RO. Food Microbiology, 2008. 3 th ed. 22-23.

[6] Ray B and Bhunia A. Fundamental of Food Microbiology, 2013. 5th ed. 61, 66, 65.

[7] Jay M, Loessner J, David A and Golden A. Modern Food Microbiology, 2006. 7th ed. 53.

[8] Pazlarová J, PhD. thesis. Factors affecting microbial growth in food.[cited 2017 April 3].available from: http://biomikro.vscht.cz/vyuka/ifm/Microbial_growth_ in_food.pdf.

[9] Barbara L, Baird-Parker, Anthony C and Grahame W. Microbiological Safety and Quality of Food, 2000.

[10] Bjorklund R, What is A Food-Borne Illness. FoodBorne Illnesses. New York: Marshall Cavendish Corporation.2005.1th ed. 10-26.

[11] Ainsworth M. Food Pathogens .Fish and Seafood Pathogens. USA. 2009.207-2018.

[12] Cheesbrough M. Microbiological examination of specimens and biochemical testing of microorganisms in Medical laboratory manual of tropical countries .Tropical Health Technology, Butterworth Heinemann Ltd. Printed in Great Britain At University Press, Cambridge. 1984. 1st ed. Vol 2. pp. 26-39, 57-69.

[13] Pitt JI, AD. Hocking, R.A. Samson and A.D. King.Recommended Methods for the Mycological Examination of Foods, In: Modern Methods in Food Mycology .Elsevier Science Ltd, Amsterdam, 1992.pp: 388, ISBN: 0444889396. 
[14] Samson R, Hoekstra E and Oorschot C. Chapter1, Introduction To Food-Born Fungi, Delft - Holland, 1981. 1-200.

[15] Ogundare AO and Adetuyi FC. Studies on the microbial population of bread baked with wheat flour from south western Nigeria. Food, Agriculture \& Environment .2003.1(3,4): 85-87.

[16] Annan-Prah A, Amewowor DHAK, Osei-Kofi J, Amoono SE, Akorli SY, Saka E and Ndadi HA. Street Foods: Handling, hygiene and client expectations in a World Heritage Site Town, Cape Coast, Ghana. African Journal of Microbiology Research.2011; 5(13):1629-1634.

[17] Oranusi S, Omagbemi F and Eni AO. Microbiological safety evaluation of snacks sold in fast food shops in Ota, Ogun state, Nigeria. International Journal Of Agricultural and Food Science. 2011 1(4): 75-79.

[18] Marzoq HY, Saeed ZF and Hasan RN. Isolation and identification of some pathogenic bacteria and fungi from restaurants and meat shops in Al-Samawa City.J.Thi-Qar Sci. 2013. 4(1):78-83.

[19] Turblin V. microbiological tools for quality assurance in hatchery: Laboratory Methods. CEVA Animal Health Asia Pacific .Malaysia.available, 2019 (29).

[20]Easa S. The microbial quality of fast food and traditional fast food .Nature and Science.Cairo, Eygypt.2010; 8(10). 